\title{
Robust reconstruction of a signal from its unthresholded recurrence plot subject to disturbances
}

Citation for published version (APA):

Sipers, A., Borm, P., \& Peeters, R. (2017). Robust reconstruction of a signal from its unthresholded recurrence plot subject to disturbances. Physics Letters A, 381(6), 604-615.

https://doi.org/10.1016/j.physleta.2016.12.028

Document status and date:

Published: 12/02/2017

DOI:

10.1016/j.physleta.2016.12.028

Document Version:

Publisher's PDF, also known as Version of record

Document license:

Taverne

Please check the document version of this publication:

- A submitted manuscript is the version of the article upon submission and before peer-review. There can be important differences between the submitted version and the official published version of record.

People interested in the research are advised to contact the author for the final version of the publication, or visit the DOI to the publisher's website.

- The final author version and the galley proof are versions of the publication after peer review.

- The final published version features the final layout of the paper including the volume, issue and page numbers.

Link to publication

\footnotetext{
General rights rights.

- You may freely distribute the URL identifying the publication in the public portal. please follow below link for the End User Agreement:

www.umlib.nl/taverne-license

Take down policy

If you believe that this document breaches copyright please contact us at:

repository@maastrichtuniversity.nl

providing details and we will investigate your claim.
}

Copyright and moral rights for the publications made accessible in the public portal are retained by the authors and/or other copyright owners and it is a condition of accessing publications that users recognise and abide by the legal requirements associated with these

- Users may download and print one copy of any publication from the public portal for the purpose of private study or research.

- You may not further distribute the material or use it for any profit-making activity or commercial gain

If the publication is distributed under the terms of Article $25 \mathrm{fa}$ of the Dutch Copyright Act, indicated by the "Taverne" license above, 


\title{
Robust reconstruction of a signal from its unthresholded recurrence plot subject to disturbances
}

\author{
Aloys Sipers ${ }^{\mathrm{a}, *}$, Paul Borm ${ }^{\mathrm{a}}$, Ralf Peeters ${ }^{\mathrm{b}}$ \\ a Department of Bèta Sciences and Technology, Zuyd University, The Netherlands \\ ${ }^{\mathrm{b}}$ Department of Data Science and Knowledge Engineering, Maastricht University, The Netherlands
}

\section{A R T I C L E I N F O}

\section{Article history:}

Received 14 March 2016

Received in revised form 10 December 2016

Accepted 12 December 2016

Available online 15 December 2016

Communicated by C.R. Doering

\section{Keywords:}

Recurrence plot

Graph theory

Robust reconstruction

Disturbances

Singular value decomposition

\begin{abstract}
A B S T R A C T
To make valid inferences from recurrence plots for time-delay embedded signals, two underlying key questions are: (1) to what extent does an unthresholded recurrence (URP) plot carry the same information as the signal that generated it, and (2) how does the information change when the URP gets distorted. We studied the first question in our earlier work [1], where it was shown that the URP admits the reconstruction of the underlying signal (up to its mean and a choice of sign) if and only if an associated graph is connected. Here we refine this result and we give an explicit condition in terms of the embedding parameters and the discrete Fourier spectrum of the URP. We also develop a method for the reconstruction of the underlying signal which overcomes several drawbacks that earlier approaches had. To address the second question we investigate robustness of the proposed reconstruction method under disturbances. We carry out two simulation experiments which are characterized by a broad band and a narrow band spectrum respectively. For each experiment we choose a noise level and two different pairs of embedding parameters. The conventional binary recurrence plot (RP) is obtained from the URP by thresholding and zero-one conversion, which can be viewed as severe distortion acting on the URP. Typically the reconstruction of the underlying signal from an RP is expected to be rather inaccurate. However, by introducing the concept of a multi-level recurrence plot (MRP) we propose to bridge the information gap between the URP and the RP, while still achieving a high data compression rate. We demonstrate the working of the proposed reconstruction procedure on MRPs, indicating that MRPs with just a few discretization levels can usually capture signal properties and morphologies more accurately than conventional RPs.
\end{abstract}

(c) 2016 Elsevier B.V. All rights reserved.

\section{Introduction}

One of the well-established techniques for analyzing a complex dynamical system is the recurrence plot (RP), see [2]. For a dynamical system a recurrence is said to occur whenever its trajectory in phase space is (nearly) self-intersecting. Examination of the recurrence pattern that occurs over an observed time interval, often provides useful information - not just when trajectories are somehow smooth and well predictable, but also in the case of complex dynamics and chaos. In these cases, recurrence quantification analysis (RQA), see [3], offers various tools to extract such information from RPs.

RPs can also be used to analyze an observed scalar signal $x(t)$. To do so, it is common to first embed it into some $M$-dimensional

\footnotetext{
* Corresponding author.

E-mail addresses: aloys.sipers@zuyd.nl (A. Sipers), paul.borm@zuyd.nl (P. Borm), ralf.peeters@maastrichtuniversity.nl (R. Peeters).
}

space by constructing a vector of time-delayed copies $X(t):=$ $(x(t), x(t+\tau), \ldots, x(t+(M-1) \tau))^{T}$. This is known as the timedelay embedding method, first introduced in [4]. Note that the vector $X(t)$ carries information on the recent history of the signal $x(t)$ and captures its local morphology. In the literature there exist several other modeling and signal processing techniques, such as AR-modeling [5] and singular spectral analysis [6], which also interpret $X(t)$ as a state vector of the dynamical process generating $x(t)$, but we will not go into them here.

The unthresholded recurrence plot (URP) is defined as the bivariate distance function $\operatorname{URP}_{X}(u, v):=\|X(u)-X(v)\|$, for some chosen norm $\|\cdot\|$. From it, the (binary) RP is obtained by choosing a threshold value $\varepsilon>0$ and defining $\operatorname{RP}_{X}^{\varepsilon}(u, v):=\Theta\left(\varepsilon-\operatorname{URP}_{X}(u, v)\right)$. Here $\Theta(\cdot)$ denotes the Heaviside step function, given by $\Theta(x)=1$ for $x \geq 0$ and $\Theta(x)=0$ for $x<0$. By construction, $\operatorname{RP}_{X}^{\varepsilon}(u, v)=1$ (and it is zero otherwise) if and only if the vectors $X(u)$ and $X(v)$ differ by at most $\varepsilon$, as measured by the norm $\|\cdot\|$. This qualifies $X(v)$ as a near recurrence of $X(u)$ and motivates the terminology used. 
When inferences are made about a signal $x(t)$ from its RP, it is important to understand to what extent an RP carries information that is unique for the underlying signal. This has been studied by several authors; see, e.g., [7], [8] and [9]. In [1], we have investigated this question in detail for the URP of a time-delay embedded signal. There we used the Euclidean norm to define the URP and we restricted the discussion to zero mean periodic signals which admit a Fourier series representation and have finite power. We will do the same in the present paper. This is a fairly large class of signals which includes many signals encountered in practice, such as digitally sampled measurement signals. The zero mean requirement is natural, as the URP does not carry any information on the mean of $x(t)$. It is easy to see that it does not carry information on the sign of $x(t)$ either. Periodicity (with a known period, which we normalize to be 1 ) allows us to deal with finite interval effects in a convenient way.

In [1, Theorem 3.4] we characterized uniqueness of the underlying signal $x(t)$ for a given URP (up to a sign \pm 1 ) by means of connectedness of a simple undirected graph $G_{X}=\left(K_{X}, E\right)$. For this graph, the node set $K_{X}$ corresponds to the discrete Fourier frequencies that occur in the signal $x(t)$. The edge set $E$ is determined by the choice of embedding dimension $M$ and the time-delay $\tau$. It was found that the (discrete Fourier) power spectrum of $x(t)$ can always be reconstructed from $\mathrm{URP}_{X}$, but it generally depends on the embedding parameters $M$ and $\tau$ which other information can also be retrieved. Several special cases were analyzed, see [1, Corollary 3.5]. However, the approach has two main shortcomings: (1) The characterization of unique reconstructibility of $x(t)$ from $\mathrm{URP}_{X}$ (up to a sign) by connectedness of $G_{X}$, is implicit. An explicit characterization in terms of $K_{X}, M$ and $\tau$ is lacking for the general case but would be useful to have: to be able to assess whether a given URP is maximally informative (i.e., the URP determines the underlying de-averaged signal up to a sign) about its underlying signal, and to help selecting embedding parameters $M$ and $\tau$ to ensure such a property when generating URPs and RPs. (2) If $G_{X}$ is connected, then the actual reconstruction of $x(t)$ from $\operatorname{URP}_{X}$ in [1] is not very practical - nor recommendable from a computational point of view. It involves several double integrals which may be hard to compute accurately, and in general it requires the search for suitable paths in the graph $G_{X}$ to connect selected nodes.

In Section 2 we address and resolve the first issue. We present a new and explicit characterization of connectedness of $G_{X}$ and, in the course of deriving it, we establish that the diameter of a connected graph $G_{X}$ always equals 1 or 2 . This new characterization shows for each choice of $M$ precisely which values of $\tau$ will cause disconnectedness. For such values, the URP will not be maximally informative and in choosing the embedding parameters we typically want to avoid them. We illustrate this result with a couple of examples.

In Section 3 we address the second issue. The 2D-Fourier transform of $U_{R P}^{2}$ is found to have coefficients with a special structure that can be exploited to compute $x(t)$. The coefficients are used to calculate a square matrix $\tilde{I}_{X}$ which is the Hadamard (i.e., entrywise) product $\tilde{I}_{X}=W \odot T$ of a rank-one matrix $W$ (determined by the non-zero Fourier coefficients of $x(t)$ only) and a known matrix $T$ (depending entirely on $M$ and $\tau$ ). The matrix $T$ allows for the determination of connectedness of $G_{X}$. If connectedness holds, then, using the property that the diameter is at most $2, T$ can be factored from $\tilde{I}_{X}$ to obtain $W$. With singular value decomposition (SVD), the Fourier coefficients of the signal $x(t)$ can then be computed from $W$ in a robust way.

In Section 4 we present a couple of simulation experiments to demonstrate this procedure. We analyze robustness against noise of the reconstruction procedure, by adding noise of a few levels to a URP before reconstruction. We also investigate sensitivity of the reconstruction near values of $\tau$ for which disconnectedness of $G_{X}$ occurs.

Third, in Section 5 we perform experiments to study the impact of truncation on URPs, such as applied when computing RPs. This fosters the idea of using 'multi-level recurrence plots' (MRPs) instead of RPs, preserving more information while still achieving a high compression rate compared to URPs.

Section 6 concludes the paper, with a discussion of the results obtained. All the proofs are collected in Appendix A.

\section{Connectedness of the graph $G_{X}$}

We consider the class of real zero mean periodic signals $x(t)$ with period 1 , which are square integrable on $[0,1)$. Any such signal admits a Fourier series representation, see [10, Sect. 4.26], denoted by:

$x(t)=\sum_{k \in \mathbb{Z}} c_{k} e^{2 \pi k t i}$.

As the mean is zero $c_{0}=0$, and as $x(t)$ is real the complex-valued Fourier coefficients $c_{k}$ satisfy $c_{-k}=\overline{c_{k}}$ for all $k \in \mathbb{Z}$. Square integrability gives $\sum_{k \in \mathbb{Z}}\left|c_{k}\right|^{2}<\infty$. Convergence of the Fourier series on the right hand side to the function $x(t)$ happens in the $L^{2}$-sense; it happens pointwise under additional smoothness conditions, see [10, Ch. 5]. Note that discrete-time (regularly sampled) signals with a finite number of observations can also be included in this set-up, as they admit finite Fourier series representations and can be associated with continuous-time periodic signals through interpolation and periodic extension.

For a given choice of embedding dimension $M$ and time-delay $\tau \in(0,1)$, let $X(t)$ be the corresponding time-delay embedded trajectory in $\mathbb{R}^{M}$ and $\operatorname{URP}_{X}(u, v)=\|X(u)-X(v)\|$ the unthresholded recurrence plot, in which $\|\cdot\|$ denotes the Euclidean norm. Note that $X(t)$ is also periodic with period 1 and given by:

$X(t)=\sum_{k \in \mathbb{Z}} c_{k} e^{2 \pi k t i} T_{k}$,

in which

$T_{k}=\left(\begin{array}{c}1 \\ z^{k} \\ \vdots \\ z^{(M-1) k}\end{array}\right), \quad$ with $z=e^{2 \pi \tau i}$.

As in [1], the associated (simple, undirected) graph $G_{X}$ is defined to have the integer labeled nodes $K_{X}=\left\{k \in \mathbb{Z} \mid c_{k} \neq 0\right\}$. Two distinct nodes $p, q \in K_{X}$ are defined to be adjacent if and only if the $L^{2}$-inner product $\left\langle T_{p}, T_{q}\right\rangle$ is nonzero. The latter is equivalently characterized by $[(p-q) \tau \in \mathbb{Z}] \vee[(p-q) M \tau \notin \mathbb{Z}]$; see [1, Lemma 3.1].

From [1, Theorem 3.4], we have that the signal $x(t)$ can be reconstructed (up to a sign \pm 1 ) from URP $X$ if and only if the graph $G_{X}$ is connected. In [1, Corollary 3.5] several special cases are addressed for which it is possible to quickly decide on connectedness of the graph $G_{X}$. Below, we extend these results by providing a complete and explicit characterization of connectedness of $G_{X}$. We also show that if $G_{X}$ is connected, then the diameter of $G_{X}$ is either 1 or 2 . This means that a connected graph $G_{X}$ is either complete (diam $\left(G_{X}\right)=1$ ) or every two nodes are connected by a path of length at most 2 .

Theorem 2.1. Let $x(t)$ be a non-zero signal from the class introduced above, and $G_{X}=\left(K_{X}, E\right)$ the associated graph for a given choice of embedding dimension $M$ and time-delay $\tau \in(0,1)$. For the associated set of node indices $K_{X}$, define the set of ordered node pairs as 
$P=\left\{(p, q) \mid p, q \in K_{X}, p>q\right\}$ and the set of absolute differences as $D=\{p-q \mid(p, q) \in P\}$. Define $h=G C D(D)$ to be the greatest common divisor of the values in $D$. Then:

(1) $G_{X}$ is connected if and only if $[h \tau \in \mathbb{Z}] \vee[h M \tau \notin \mathbb{Z}]$.

(2) If $G_{X}$ is connected, then $\operatorname{diam}\left(G_{X}\right) \in\{1,2\}$.

Remark 1. Note that the characterization of connectedness of $G_{X}$ by statement (1) of Theorem 2.1, closely resembles the definition of adjacency of two nodes $p$ and $q$. If $G_{X}$ has exactly two nodes, then the set $P$ consists of a single node pair $(p, q)$ and the set $D$ contains a single value, whence $h=p-q$. Then the two characterizations coincide. When more node pairs are involved, the theorem shows that $h$ plays the same role in describing connectedness of the graph as $p-q$ does for adjacency of individual nodes.

Remark 2. If it is found that for some choice of the embedding parameters $M$ and $\tau$ the URP of a time-delay embedded signal is not maximally informative, then $h M \tau \in \mathbb{Z}$ and $h \tau \notin \mathbb{Z}$. Then leaving $\tau$ unchanged and altering the embedding dimension to $M+1$ or $M-1$ will produce URPs which are. The same holds when $M$ is left unchanged but $\tau$ is replaced by $\tau\left(1+\frac{1}{M}\right)$ or $\tau\left(1-\frac{1}{M}\right)$.

Remark 3. The value $h$ relates to periodicities in $x(t)$ and in $\mathrm{URP}_{X}$. Denote $m=\min \left\{k \in K_{X} \mid k>0\right\}$. Recall that also $-m \in K_{X}$, so that $2 m \in D$ and $h$ divides $2 m$. It is not hard to show that if $h$ divides $m$ then $h$ divides all $k \in K_{X}$ and $x\left(t+\frac{1}{h}\right)=x(t)$. In this case $U_{R P}$ has a corresponding $h \times h$ 2D-periodic pattern. If $h$ does not divide $m$ then instead it holds that $x\left(t+\frac{1}{h}\right)=-x(t)$, whence $x\left(t+\frac{2}{h}\right)=x(t)$. In this case URP $X_{X}$ has a corresponding $h \times h$ checkerboard pattern as well as a $\frac{h}{2} \times \frac{h}{2} 2 \mathrm{D}$-periodic pattern.

In Fig. 1, three examples of periodic signals are given to illustrate Theorem 2.1. Each signal is analyzed for two different settings of the embedding parameters $M$ and $\tau$. In all cases the signal has a power spectrum with 2 different frequencies, so $G_{X}$ has 4 nodes. In none of these cases connectedness of $G_{X}$ can be analyzed with [1, Corollary 3.5].

In Fig. $1(\mathrm{i})-(\mathrm{v})$ the signal is $x(t)=2 \sin (6 \pi t)+\cos (6 \pi t)-$ $\cos (12 \pi t)+\sin (12 \pi t)$, which has $K_{X}=\{-6,-3,3,6\}$, with $m=3$ and $h=3$. As $h$ divides $m$, it holds that $x\left(t+\frac{1}{3}\right)=x(t)$ and therefore $\operatorname{URP}_{X}(u, v)$ displays a $3 \times 3$ periodic pattern, with $\operatorname{URP}_{X}(u+$ $\left.\frac{1}{3}, v\right)=\operatorname{URP}_{X}(u, v)$ as well as $\operatorname{URP}_{X}\left(u+\frac{1}{3}, v+\frac{1}{3}\right)=\operatorname{URP}_{X}(u, v)$. For $M=4$ and $\tau=\frac{1}{24}$ the graph $G_{X}$ is connected, because $h M \tau=$ $\frac{1}{2} \notin \mathbb{Z}$. For $M=4$ and $\tau=\frac{1}{6}$ the graph $G_{X}$ is disconnected, since $h \tau=\frac{1}{2} \notin \mathbb{Z}$ and $h M \tau=2 \in \mathbb{Z}$.

In Fig. 1 (vi) $-(\mathrm{x})$ the signal is $x(t)=2 \sin (6 \pi t)+\cos (6 \pi t)-$ $\cos (10 \pi t)+\sin (10 \pi t)$, which has $K_{X}=\{-5,-3,3,5\}$, with $m=3$ and $h=2$. Now $h$ does not divide $m$, so it holds that $x\left(t+\frac{1}{2}\right)=$ $-x(t)$ and therefore $\operatorname{URP}_{X}(u, v)$ displays a $2 \times 2$ checkerboard pattern, with $\operatorname{URP}_{X}\left(u+\frac{1}{2}, v+\frac{1}{2}\right)=\operatorname{URP}_{X}(u, v)$. For $M=4$ and $\tau=\frac{1}{16}$ the graph $G_{X}$ is connected, because $h M \tau=\frac{1}{2} \notin \mathbb{Z}$. For $M=4$ and $\tau=\frac{1}{8}$ the graph $G_{X}$ is disconnected, since $h \tau=\frac{1}{4} \notin \mathbb{Z}$ and $h M \tau=1 \in \mathbb{Z}$.

In Fig. $1(x i)-(x v)$ the signal is $x(t)=2 \sin (6 \pi t)-\cos (6 \pi t)+$ $\cos (18 \pi t)+\sin (18 \pi t)$, which has $K_{X}=\{-9,-3,3,9\}$ with $m=3$ and $h=6$. Again $h$ does not divide $m$, and now it holds that $x(t+$ $\left.\frac{1}{6}\right)=-x(t)$ and therefore $\operatorname{URP}_{X}(u, v)$ displays a $6 \times 6$ checkerboard pattern, with $\operatorname{URP}_{X}\left(u+\frac{1}{6}, v+\frac{1}{6}\right)=\operatorname{URP}_{X}(u, v)$. Because $x\left(t+\frac{1}{3}\right)=$ $x(t)$, it also displays a $3 \times 3$ periodic pattern. For $M=4$ and $\tau=\frac{1}{6}$ the graph $G_{X}$ is connected, because $h \tau=1 \in \mathbb{Z}$. For $M=4$ and $\tau=\frac{1}{8}$ the graph $G_{X}$ is disconnected, since $h \tau=\frac{3}{4} \notin \mathbb{Z}$ and $h M \tau=$ $3 \in \mathbb{Z}$. In this case the URP has in fact a $6 \times 6$ periodic pattern.

From Theorem 2.1 it follows for a signal $x(t)$ with a given frequency content (i.e., with $K_{X}$ known) and for a given embedding dimension $M$, at which values of $\tau$ disconnectedness of $G_{X}$ occurs. At such $\tau$ the reconstruction of $x(t)$ up to a sign \pm 1 from its URP is non-unique, implying that the URP is not maximally informative about its underlying signal $x(t)$. In selecting a good value for $\tau$, we want to stay away from such values. Sensitivity analysis at those values will indicate to which extent ill-conditioning occurs in close neighborhoods, allowing us to search for locations which are better behaved.

The value of $h$ depends entirely on $K_{X}$ and not on the embedding parameters $M$ and $\tau$. Note that 'generically' $h=1$, for which the values of $\tau$ at which disconnectedness occurs are precisely the integer multiples of $\frac{1}{M}$. If the power spectrum of $x(t)$ has most of its energy concentrated at a subset of indices in $K_{X}$ for which the GCD of their differences is larger than one, then in view of robustness it is important to be careful and to take the corresponding value $\hat{h}$ of this GCD into account (in the role of $h$ ). This suggests to start computing the GCD from the values of $k,-k \in K_{X}$ which correspond to the Fourier coefficients having the highest energy (and possibly using a threshold strategy for robustness). The GCD computed from such a subset of $K_{X}$ will provide an upper bound $\hat{h}$ on $h$, while $h$ divides $\hat{h}$. Then one may decide to let $\tau$ stay away from all the integer multiples of $\frac{1}{\hat{h} M}$, which will be an enveloping set for the true values at which disconnectedness occurs.

For a discrete-time sampled signal with $N$ measured observations, a corresponding continuous-time signal $x(t)$ can be constructed to have a finite Fourier series with $k \in\{-K, \ldots, K\}$ where $K=\left\lfloor\frac{N}{2}\right\rfloor$. Then it is natural to take $\tau$ to be an integer multiple of $\frac{1}{N}$, to facilitate the time-delay embedding by shifting the measurements without interpolation. It now becomes important to select such a value for $\tau$ which stays well away from the integer multiples of $\frac{1}{h M}$, which is not a hard problem to analyze efficiently.

\section{Reconstruction of $x(t)$ from its URP}

In [1, Section 5] a procedure was described to reconstruct a signal from its URP. Though valid for theoretical purposes, it is not recommendable for practical use as it has several drawbacks. For instance, it involves the computation of multiple double integrals which is numerically cumbersome and unattractive. It also requires, for every pair of nodes $(p, q)$ in the graph $G_{X}$, the selection of a connecting path, for which it is unclear how to obtain one efficiently and how it will affect the numerical accuracy of the calculations. In this section we present a novel method for reconstructing $x(t)$ from $\operatorname{URP}_{X}(u, v)$ which aims to overcome these drawbacks. It uses 2D-Fourier series representations and singular value decomposition and it employs part (2) of Theorem 2.1 to simultaneously involve all paths of length 2 to connect a node pair $(p, q)$.

We start by recalling the result of [1, Lemma 3.1], which states that the $L^{2}$-inner product of the vectors $T_{p}$ and $T_{q}$ is explicitly given by:

$$
\left\langle T_{p}, T_{q}\right\rangle= \begin{cases}M & \text { for }(p-q) \tau \in \mathbb{Z}, \\ \frac{\sin (\pi(p-q) M \tau)}{\sin (\pi(p-q) \tau)} e^{\pi(p-q)(M-1) \tau i} & \text { for }(p-q) \tau \notin \mathbb{Z} .\end{cases}
$$

Clearly its value depends only on the difference $p-q$, whence $\left\langle T_{p}, T_{q}\right\rangle=\left\langle T_{p-q}, T_{0}\right\rangle$.

In [1] it was shown that $\operatorname{URP}_{X}(u, v)$ carries the same information as the inner product $I_{X}(u, v):=\langle X(u), X(v)\rangle$, both regarded as periodic two-variable functions of $u, v \in[0,1)$. We can represent $I_{X}(u, v)$ by its 2D-Fourier series representation:

$I_{X}(u, v)=\sum_{p \in \mathbb{Z}} \sum_{q \in \mathbb{Z}} I_{X, p, q} e^{2 \pi(p u+q v) i}$, 


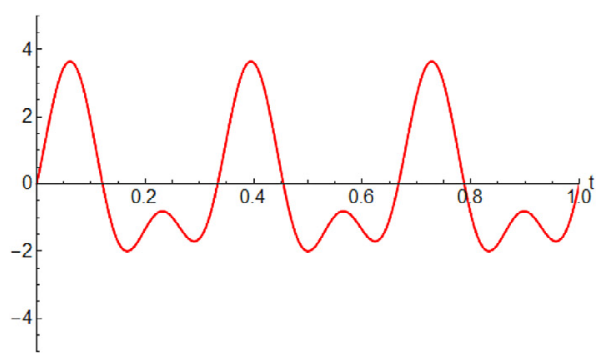

(i) Periodic signal $x(t)$ with $K_{X}=\{-6,-3,3,6\}$,

$$
h=3 \text {. }
$$

$$
M=4, \tau=\frac{1}{6}
$$

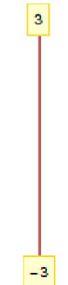

(ii) $G_{X}$ is disconnected.

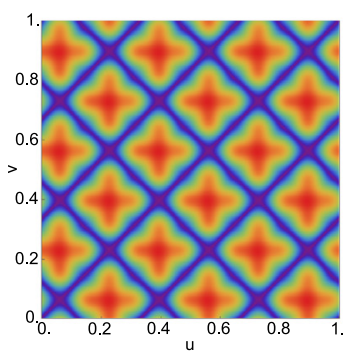

(iii) URP with a $3 \times 3$ periodic pattern.

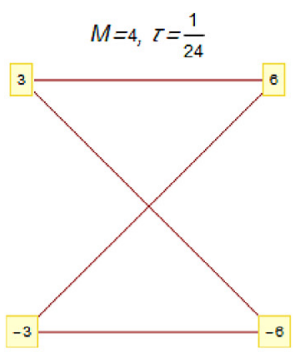

(iv) $G_{X}$ is connected.

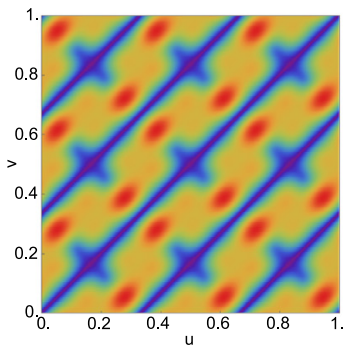

(v) URP with a $3 \times 3$ periodic pattern.

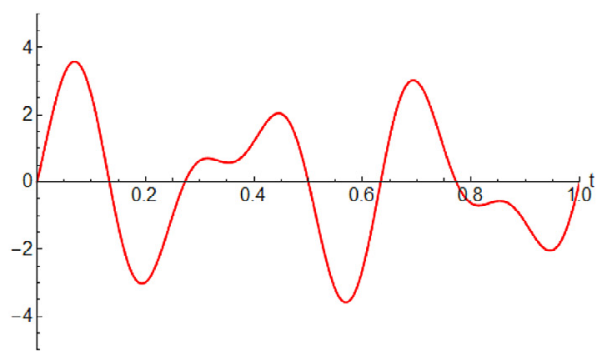

(vi) Periodic signal $x(t)$ with $K_{X}=\{-5,-3,3,5\}$,

$$
h=2 \text {. }
$$$$
M=4, I=\frac{1}{8}
$$

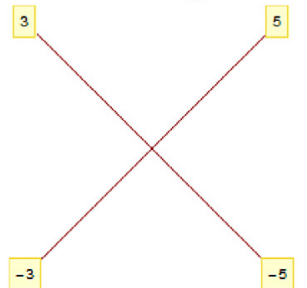

(vii) $G_{X}$ is disconnected.

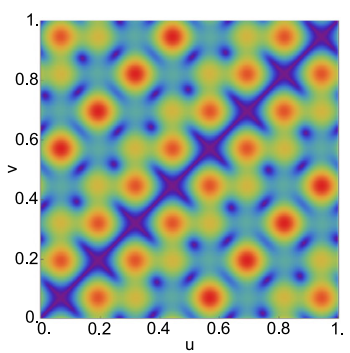

(viii) URP with a $2 \times 2$ checkerboard pattern.

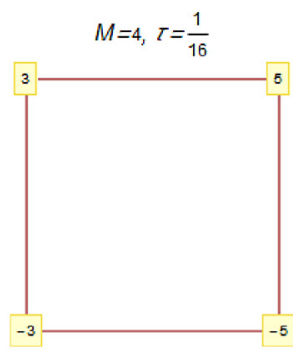

(ix) $G_{X}$ is connected.

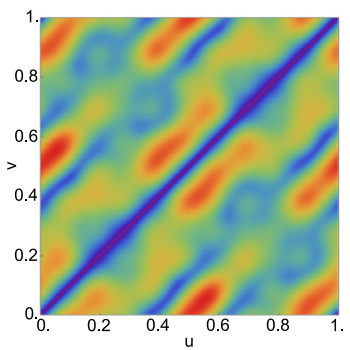

(x) URP with a $2 \times 2$ checkerboard pattern.

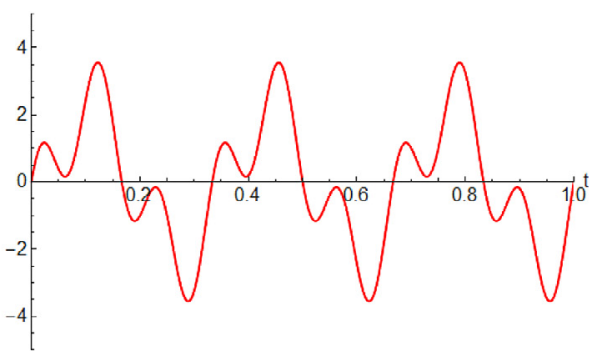

(xi) Periodic signal $x(t)$ with $K_{X}=\{-9,-3,3,9\}$, $h=6$.

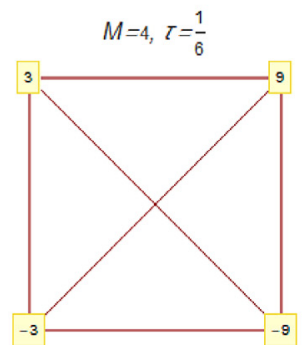

(xii) $G_{X}$ is a complete graph.

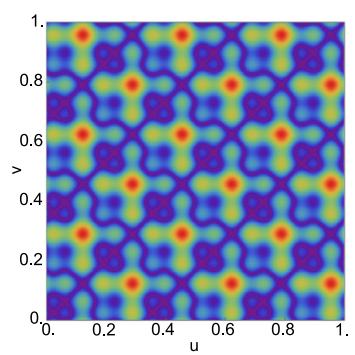

(xiii) URP: $6 \times 6$ checkerboard pattern, $3 \times 3$ periodic pattern.

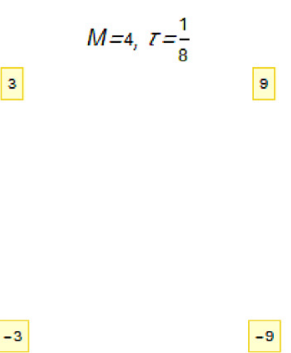

(xiv) $G_{X}$ is totally disconnected.

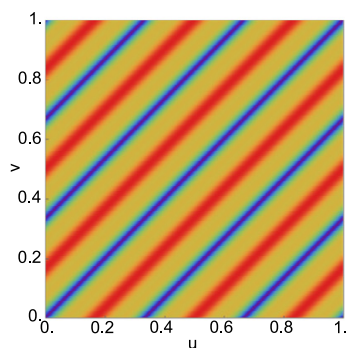

(xv) URP: $6 \times 6$ checkerboard pattern, $3 \times 3$ periodic pattern

Fig. 1. Three periodic signals, (dis)connectedness of their graphs $G_{X}$ and periodicity of their URPs, each for two different choices of embedding parameters. 
in which the 2D-Fourier coefficients are denoted by $I_{X, p, q}$. Substitution of the Fourier series representations of $X(u)$ and $X(v)$ in $\langle X(u), X(v)\rangle$ gives:

$I_{X, p, q}=c_{p} \overline{c_{-q}}\left\langle T_{p}, T_{-q}\right\rangle=c_{p} c_{q}\left\langle T_{p+q}, T_{0}\right\rangle$,

where it is used that $\overline{c_{-q}}=c_{q}$ for a real signal $x(t)$.

The squared URP, $\|X(u)-X(v)\|^{2}$, obviously has the same information content as the URP itself. Its 2D-Fourier series representation is now observed to be closely related to that of $I_{X}(u, v)$.

Proposition 3.1. Let $x(t)=\sum_{k \in \mathbb{Z}} c_{k} e^{2 \pi k t i}$ be a signal from our class. Then, for any choice of embedding parameters $M$ and $\tau$, the squared URP can be expressed as a 2D-Fourier series

$U R P_{X}^{2}(u, v)=\sum_{p \in \mathbb{Z}} \sum_{q \in \mathbb{Z}} C_{X, p, q} e^{2 \pi(p u+q v) i}$,

for which the $2 D$-Fourier coefficients $C_{X, p, q}$ are given by

$$
C_{X, p, q}=\left\{\begin{array}{l}
-2 I_{X, p, q}=-2 c_{p} \overline{c_{-q}}\left\langle T_{p+q}, T_{0}\right\rangle \\
\text { for } p \neq 0 \text { and } q \neq 0 \\
\sum_{n \in \mathbb{Z}} I_{X, p-n, n}=\left\langle T_{p}, T_{0}\right\rangle \sum_{n \in \mathbb{Z}} c_{n} \overline{c_{n-p}} \\
\quad \text { for } p \neq 0 \text { and } q=0, \\
\sum_{n \in \mathbb{Z}} I_{X, n, q-n}=\left\langle T_{q}, T_{0}\right\rangle \sum_{n \in \mathbb{Z}} c_{n} \overline{c_{n-q}} \\
\quad \text { for } p=0 \text { and } q \neq 0, \\
2 \sum_{n \in \mathbb{Z}} I_{X, n,-n}=2 M \sum_{n \in \mathbb{Z}}\left|c_{n}\right|^{2} \\
\text { for } p=0 \text { and } q=0 .
\end{array}\right.
$$

The procedure of [1, Section 5] relies on the computation of the inner product function $I_{X}(u, v)$ from $\operatorname{URP}_{X}(u, v)$. Proposition 3.1 makes clear that a more convenient direct approach is possible, because the 2D-Fourier coefficients of $\operatorname{URP}_{X}^{2}(u, v)$ give immediate access to the 2D-Fourier coefficients of $I_{X}(u, v)$ : it holds that $I_{X, p, q}=-C_{X, p, q} / 2$ for all $p, q \neq 0 .^{1}$

To complete the reconstruction of $x(t)$ from $\operatorname{URP}_{X}^{2}(u, v)$, we proceed to compute the Fourier coefficients $c_{k}$ from the coefficients $I_{X, p, q}$. From Eqn. (6) we have that

$c_{p} \overline{c_{q}}=I_{X, p,-q} /\left\langle T_{p-q}, T_{0}\right\rangle$,

provided that $\left\langle T_{p-q}, T_{0}\right\rangle \neq 0$, i.e.: if $p$ and $q$ are adjacent in $G_{X}$. If a URP has a finite 2D spectrum, then the finite-sized matrix of coefficients $W=\left(c_{p} \overline{c_{q}}\right)$ is Hermitian and of rank one, as it can be factored as

$W=w w^{*}$

where $w$ is a vector containing the Fourier coefficients $c_{k}$ of the signal $x(t)$ as its elements. This holds if $x(t)$ has a finite Fourier series representation, say $x(t)=\sum_{k=-K}^{K} c_{k} e^{2 \pi k t i}$, which for instance applies to sampled signals. Likewise, it holds in the common situation where the URP itself is available only in digital form as a finite-sized matrix; in that case a sampled signal can be considered to have generated it.

The vector $w$ is determined by the matrix $W=w w^{*}$ up to a complex unimodular scalar. From the property $c_{-k}=\overline{c_{k}}$, it follows that $w$ has additional structure which allows one to recover $w$ from $W$ up to a sign \pm 1 . Spectral decomposition as achieved by singular value decomposition (SVD) offers a robust technique for this. The following two propositions make this explicit.

\footnotetext{
1 Because $I_{X, p, 0}=I_{X, 0, q}=0$, this also makes clear that $\operatorname{URP}_{X}^{2}(u, v)$ fully determines $I_{X}(u, v)$, while the converse follows from the expressions of Proposition 3.1. This reconfirms the information equivalence of $\operatorname{URP}_{X}(u, v)$ and $I_{X}(u, v)$ by different arguments than given in [1].
}

Proposition 3.2. Let $x(t)$ be a zero-mean real signal $x(t)$ from our class, assumed to have a nonempty finite spectrum. Let $w$ be a vector containing the non-zero Fourier coefficients $c_{k}, k \in K_{X}$, in some specified order. Let $J$ be the associated permutation matrix which acts on $w$ by reversing the positions of each pair of entries $c_{k}$ and $c_{-k}$ for every $k \in K_{X}$. Define $W=w w^{*}$. Then:

(1) $J=J^{-1}=J^{T}$.

(2) $w=J \bar{w}$.

(3) $x$ is an eigenvector of $W$ if and only if $J \bar{x}$ is an eigenvector of $W$ with the same eigenvalue.

Proposition 3.3. Let $W=w w^{*}$ be a nonzero Hermitian matrix as in Proposition 3.2, with $w$ containing the nonzero Fourier coefficients $c_{k}$ of the real signal $x(t)$ in some specified order, and $J$ the associated permutation matrix reversing each pair of coefficients $c_{k}$ and $c_{-k}$. Let the spectral decomposition of the matrix $W$ be given by $W=\lambda u u^{*}$, where $\lambda>0$ is the positive real eigenvalue of $W$ and $u$ a corresponding eigenvector of unit norm. Then

$w= \pm\left(\lambda u^{*} J \bar{u}\right)^{1 / 2} u$

which specifies $w$ up to a sign \pm 1 .

Proposition 3.3 makes clear that the Fourier coefficients $c_{k}$ of $x(t)$ can all be computed, up to a joint sign \pm 1 , directly from the SVD of the matrix $W$. The matrix $W$ has rank one and is Hermitian, so its singular vectors coincide with its eigenvectors, and what is needed to find $w$ is a normalized singular vector $u$ corresponding to the only non-zero singular value. The SVD achieves this in a numerically robust way. The rank-one property of $W$ can be exploited by numerical methods to help to improve accuracy, and it implies that the non-zero eigenvalue (singular value) $\lambda$ of $W$ equals trace $\left(w w^{*}\right)=\sum_{k \in \mathbb{Z}}\left|c_{k}\right|^{2}=\int_{t=0}^{1} x(t)^{2} d t$ which is the energy of the signal $x(t)$. Using the 2D-Fourier transform of $\operatorname{URP}_{X}^{2}(u, v)$ we have $\lambda=C_{X, 0,0} /(2 M)$.

In this way, the problem of reconstructing $x(t)$ from $\operatorname{URP}_{X}(u, v)$ is reduced to the equivalent problem of computing $W$ from $\operatorname{URP}_{X}^{2}(u, v)$ or from $I_{X}(u, v)$. Eqn. (6) implies that the non-zero 2D-Fourier coefficients $I_{X, p,-q}$ can be collected in a matrix $\tilde{I}_{X}$ which can be factored as:

$\tilde{I}_{X}=W \odot T$.

Here $T$ has the entries $T_{p, q}=\left\langle T_{p-q}, T_{0}\right\rangle=\left\langle T_{p}, T_{q}\right\rangle$ given by Eqn. (4) and $\odot$ denotes the Hadamard (i.e., entrywise) matrix product. The matrix $T$ depends solely on the embedding parameters $M$ and $\tau$ and carries all the connectivity information on the graph $G_{X}$. If $G_{X}$ happens to be a complete graph, then all the entries of $T$ are non-zero and Eqn. (9) can be used to find $W$. Otherwise $T_{p, q}=0$ for some nodes $p$ and $q$, whence $I_{X, p,-q}=0$ and $W_{p, q}=c_{p} \overline{c_{q}}$ cannot be computed directly. But from part (2) of Theorem 2.1 we have that the diameter of a connected graph $G_{X}$ is either 1 or 2 . This is exploited in the following theorem.

Theorem 3.4. Let $x(t)$ be a zero-mean real signal from our class, assumed to have a nonempty finite spectrum. Let the embedding parameters $M$ and $\tau$ be given and let the matrices $\tilde{I}_{X}, W$ and $T$ be defined as described above. Define the non-negative matrix $S$ to have the entries

$S_{p, q}=\left|T_{p, q}\right|= \begin{cases}M & \text { for }(p-q) \tau \in \mathbb{Z}, \\ \frac{|\sin (\pi(p-q) M \tau)|}{|\sin (\pi(p-q) \tau)|} & \text { for }(p-q) \tau \notin \mathbb{Z} .\end{cases}$

Also, define the matrix $\hat{I}_{X}$ by $\hat{I}_{X}:=W \odot S$. Then it holds that

$M \hat{I}_{X} \operatorname{diag}\left(\hat{I}_{X}\right)^{-1} \hat{I}_{X}=W \odot S^{2}$, 
in which $\operatorname{diag}\left(\hat{I}_{X}\right)$ denotes the diagonal matrix coinciding with $\hat{I}_{X}$ on its main diagonal.

The matrix $S^{2}$ is strictly positive if and only if the graph $G_{X}$ is connected.

Note that $S$ and $\hat{I}_{X}$ can be computed from $T$ and $\tilde{I}_{X}$. The entries on the main diagonal of $\tilde{I}_{X}$ are given by $I_{X, p,-p}=M\left|c_{p}\right|^{2}$, which all are real and strictly positive (for $p \in K_{X}$ ). They coincide with the entries of $\operatorname{diag}\left(\hat{I}_{X}\right)$. As indicated, for a connected graph $G_{X}$ the matrix $S^{2}$ is strictly positive and the matrix $W$ can then be computed by elementwise division of $M \hat{I}_{X} \operatorname{diag}\left(\hat{I}_{X}\right)^{-1} \hat{I}_{X}$ by $S^{2}$ :

$W_{p, q}=M\left(\hat{I}_{X} \operatorname{diag}\left(\hat{I}_{X}\right)^{-1} \hat{I}_{X}\right)_{p, q} /\left(S^{2}\right)_{p, q}$.

Summary of the procedure to reconstruct a signal from a given URP, with known $M$ and $\tau$ :

First one computes the 2D-FFT of the squared URP, $\operatorname{URP}_{X}^{2}(u, v)$. This gives the 2D-Fourier coefficients $C_{X}$, from which the coefficients $I_{X, p, q}$ are directly obtained by Proposition 3.1. The coefficients $\tilde{I}_{X, p, q}=I_{X, p,-q}$ follow by relabeling. The node set $K_{X}$ consists of all $k$ for which $\tilde{I}_{X, k, k}=M\left|c_{k}\right|^{2}$ is positive. (For practical purposes, a numerical tolerance can be set to decide on significant deviation from zero.) To verify unique reconstructibility of $x(t)$ from $U_{R P}$ (up to a sign), one may compute $h$ from $K_{X}$ and use Theorem 2.1.

From $M, \tau$ and $K_{X}$, the matrices $T$ and $S$ follow using Eqns. (4) and (13) The matrix $\hat{I}_{X}$ follows from $\tilde{I}_{X}$ using $T$ and $S$. From $S$ the matrix $S^{2}$ is computed, of which all entries are strictly positive if and only if reconstructibility holds. Then $W$ follows by factoring $S^{2}$ from Eqn. (14) in Theorem 3.4, as indicated in Eqn. (15).

Finally, the SVD is used to compute the vector $w$ of nonzero Fourier coefficients $c_{k}$ of $x(t)$ (up to a sign), in accordance with Proposition 3.3, from which we finally build $x(t)$ itself.

To provide some insight about the numerical sensitivity of this entrywise division for small values of $\left(S^{2}\right)_{p, q}$, we have the following result.

Proposition 3.5. Let $\tau \in \mathbb{Q} \cap(0,1)$ be represented as $\tau=\frac{n}{d}$, with $n$ and $d$ co-prime integers such that $d \geq 2$ and $n \in\{1,2, \ldots, d-1\}$. Let $\delta$ be computed from d as:

$\delta= \begin{cases}\tan \left(\frac{\pi}{\max (d, 4)}\right) & \text { if } d \text { is even, } \\ 2 \sin \left(\frac{\pi}{2 d}\right) & \text { if } d \text { is odd. }\end{cases}$

(1) If $p$ and $q$ are two adjacent nodes in $K_{X}$, then:

$S_{p, q} \geq \delta$,

(2) If the graph $G_{X}$ is connected, then:

$\left(S^{2}\right)_{p, q} \geq \delta^{2}$.

As the proof in the appendix shows, the value of $\delta$ in this proposition refines the easier to prove lower bound $\sin \left(\frac{\pi}{d}\right)$. This improvement is particularly useful for small values of $d$. The bound in part (2) can also be used to set a threshold to test connectedness of $G_{X}$ numerically, by evaluating the entries of $S^{2}$. Typically the bounds provided here are not sharp, especially if $G_{X}$ has many nodes and each node pair $(p, q)$ is connected by many different paths of length 2 .

\section{Simulation experiments}

To investigate robustness and sensitivity of the SVD-based reconstruction procedure proposed in the previous section, we have carried out a couple of simulation experiments. In each of these experiments we start from a known signal $x(t)$, treated as a periodic signal on $[0,1]$, for which we record the frequency content. I.e., we determine the node set $K_{X}$ and also the value of $h$. Then we select embedding parameters $M$ and $\tau$, for which we determine the unthresholded recurrence plot $\operatorname{URP}_{X}(u, v)$. Next, we add a disturbance to this URP to simulate an 'observed' URP, denoted $\widehat{U R P}_{X}$, which we use to reconstruct a signal $\hat{x}(t)$ by the proposed method.

This reconstruction proceeds as described in the summary of the procedure given in the previous section, starting from $\widehat{\mathrm{URP}}_{X}$. To determine a corresponding node set $\widehat{K}_{X}$, one must use a threshold, which can be set in various ways. In our experiments we did not aim to optimize this, but simply decided to choose $\widehat{K}_{X}$ to consist of all $k$ for which $\left|\hat{c}_{k}\right|^{2}$ exceeds $0.01 \max \left\{\left|\hat{c}_{\ell}\right|^{2} \mid \ell \in \mathbb{Z}\right\}$. (Then $\hat{h}$ can be computed from $\widehat{K}_{X}$, and used to verify reconstructibility.) The approximation $\hat{x}(t)$ which finally results can then be compared to the original $x(t)$ to assess the quality of the approximation, in view of the nature of the given signal, the selected embedding parameters, and the amount of noise added to the URP.

The embedding parameters in the next two experiments are chosen such that in the cases (A) and (B) the graph $G_{X}$ is connected and disconnected respectively. For a connected graph $G_{X}$ we may observe to which extent the reconstruction gets distorted as a result of the noise we introduced. When $G_{X}$ is disconnected, reconstruction is nonunique, but this may not properly be recognized when noise is added.

Experiment 1: Reconstruction of a triangular signal with a narrow band spectrum.

The periodic signal $x(t)$ of this experiment resembles a triangular wave. It is displayed, together with its discrete spectrum, in Fig. 2. It consists of 1000 samples, it is of zero mean and it has a narrow band spectrum with 5 low frequencies only, with $K_{X}= \pm\{1,3,5,7,9\}$. The corresponding value of $h$ equals 2 . It therefore holds that $x\left(t+\frac{1}{2}\right)=-x(t)$, for all $t$.

(A) For the embedding parameters $M=4$ and $\tau=0.200$ the URP of the signal is given in Fig. 3(A). For these parameters $h M \tau=1.600 \notin \mathbb{Z}$, so that $G_{X}$ is connected and $x(t)$ is uniquely reconstructible (up to a sign) from $U R P_{X}$. The disturbance applied to this (true) URP was chosen to consist of normally distributed white noise of zero mean and a small standard deviation given by $\sigma=0.008 \max \left(\operatorname{URP}_{X}\right)$. The observed URP does not visibly differ from the true URP. The reconstructed signal $\hat{x}(t)$ is displayed in Fig. 4(B) (in blue) together with the original signal $x(t)$ (in red) and the mismatch between both (in green). The corresponding node set $\widehat{K}_{X}$ was obtained as $\widehat{K}_{X}=K_{X} \cup \pm\{112\}$ with $\hat{h}=1$.

(B) For the embedding parameters $M=4$ and $\tau=0.125$, the graph $G_{X}$ is disconnected so that unique reconstruction of $x(t)$ is impossible and the estimate $\hat{x}(t)$ will be unreliable. The resulting URP is given in Fig. 3(B). The disturbance applied to this (true) URP was again chosen to consist of normally distributed white noise of zero mean and the small standard deviation $\sigma=0.008 \max \left(\mathrm{URP}_{X}\right)$. The observed URP again does not visibly differ from the true URP. The reconstructed signal $\hat{x}(t)$ is displayed in Fig. 4(B) (in blue) together with the original signal $x(t)$ (in red, largely overlayed) and the mismatch between both (in green). The corresponding node set $\widehat{K}_{X}$ was in this case obtained as $\widehat{K}_{X}=K_{X} \cup \pm\{430\}$ for which $\hat{h}=1$. Note that $\hat{h} M \tau=0.500 \notin \mathbb{Z}$, which indicates connectedness of $G_{X}$ and unique reconstructibility of $x(t)$ during the reconstruction procedure.

We conclude that the proposed method in case (A) allows the signal $x(t)$ to be reconstructed with high accuracy from the lightly disturbed URP. In case (B), the value $\tau=0.125$ is unwanted, as it corresponds to $G_{X}$ being disconnected. The chosen threshold for determining $\widehat{K}_{X}$ in this case is too low, causing frequencies introduced by the disturbance to be included in the reconstruction and 


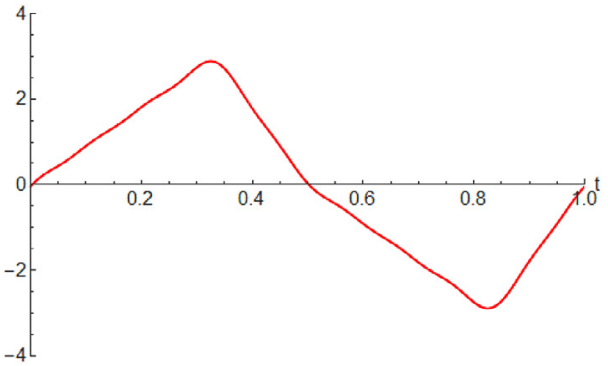

(i)

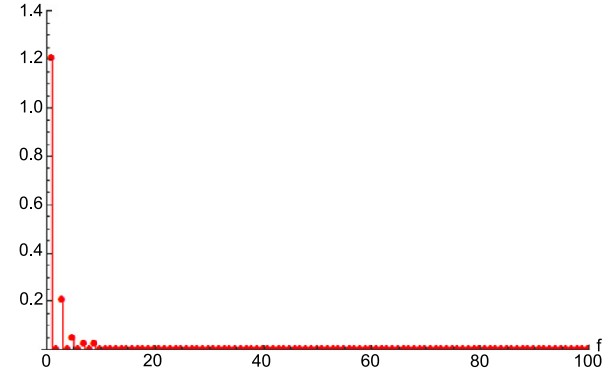

(ii)

Fig. 2. Signal $x(t)$ for Experiment 1. (i) The triangular zero mean periodic signal. (ii) Its narrow band power spectrum.

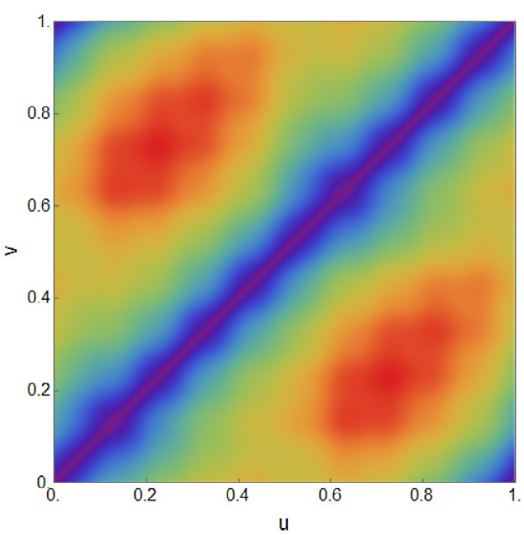

(A)

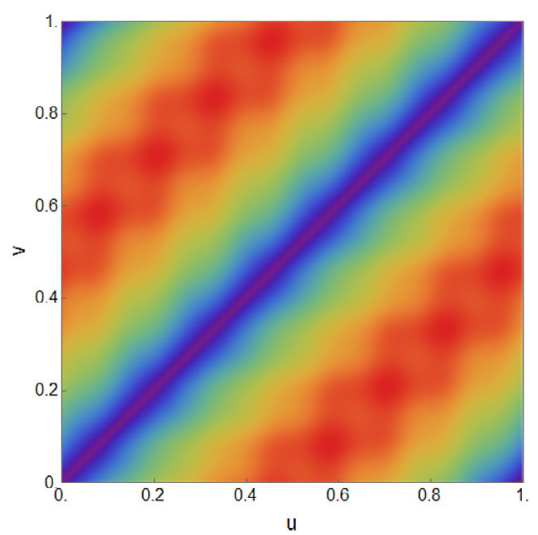

(B)

Fig. 3. URPs for $x(t)$ in Experiment 1. (A) For $M=4$ and $\tau=0.200$. (B) For $M=4$ and $\tau=0.125$.

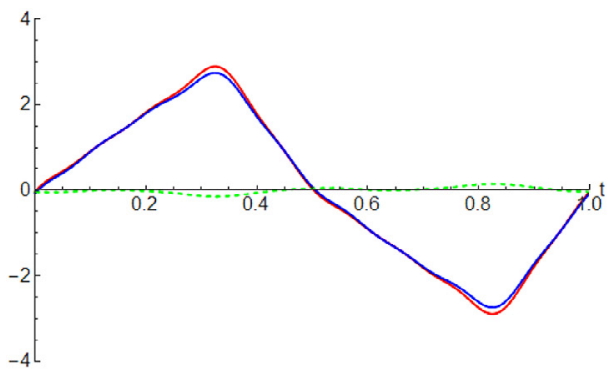

(A)

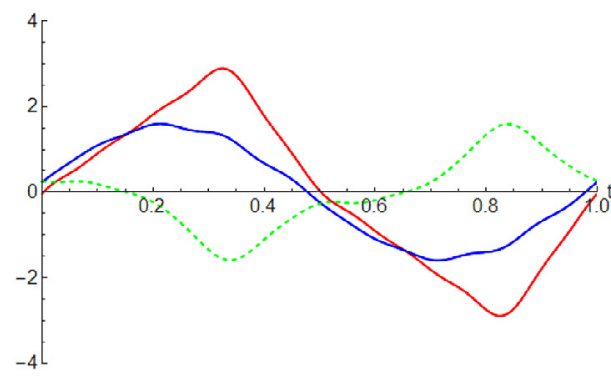

(B)

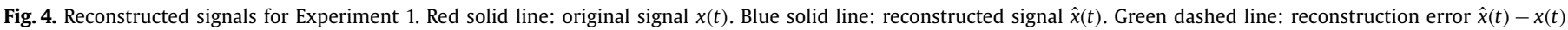
(A) For $M=4$ and $\tau=0.200$. (B) For $M=4$ and $\tau=0.125$. (For interpretation of the colors in this figure, the reader is referred to the web version of this article.)

disconnectedness of $G_{X}$ not to be recognized. The resulting approximation $\hat{x}(t)$ is inaccurate (though the URPs are highly similar). This emphasizes the need to determine $\widehat{K}_{X}$ and $\hat{h}$ more conservatively.

Experiment 2: Reconstruction of an ECG-like signal with a broad band spectrum.

The signal $x(t)$ of this experiment resembles an ECG signal. It is displayed, together with its discrete spectrum, in Fig. 5. As in the previous experiment, it consists of 1000 samples. It was filtered to have zero mean and a broad band spectrum with 45 frequencies, whence $K_{X}= \pm\{1,2, \ldots, 45\}$. The corresponding value of $h$ equals 1.

(A) For the embedding parameters $M=5$ and $\tau=0.010$ the URP of the signal is given in Fig. 6(A). For these parameters $h M \tau=0.05 \notin \mathbb{Z}$, so that $G_{X}$ is connected and $x(t)$ is uniquely reconstructible (up to a sign) from $U_{R P_{X}}$. The disturbance ap- plied to this (true) URP was chosen to consist of normally distributed white noise of zero mean and a very small standard deviation: $\sigma=0.0006 \max \left(\operatorname{URP}_{X}\right)$. The observed URP does not visibly differ from the true URP. The reconstructed signal $\hat{x}(t)$ is displayed in Fig. 7(A) (in blue) together with the original signal $x(t)$ (in red) and the mismatch between both (in green). The corresponding node set $\widehat{K}_{X}$ was obtained as $\widehat{K}_{X}=K_{X} \cup$ $\pm\{71,72,107,167,170,215,289,479,490\}$ with $\hat{h}=1$.

(B) For the embedding parameters $M=5$ and $\tau=0.200$, the graph $G_{X}$ is disconnected so that unique reconstruction of $x(t)$ is impossible and the estimate $\hat{x}(t)$ will be unreliable. Avoiding this choice of $\tau$, we set it to the nearby value $\tau=0.199$ (requiring $\tau$ to be a multiple of $1 / 1000$ to deal with delays of the sampled signal in a convenient way). The resulting URP is given in Fig. 6(B). Now $h M \tau=0.995 \notin \mathbb{Z}$, so that $G_{X}$ is again connected and $x(t)$ is uniquely reconstructible (up to a sign) from $U R P_{X}$. The disturbance applied to this (true) URP was again chosen to consist of normally 


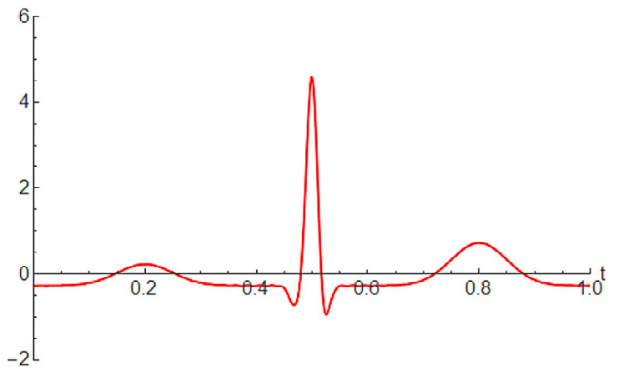

(i)

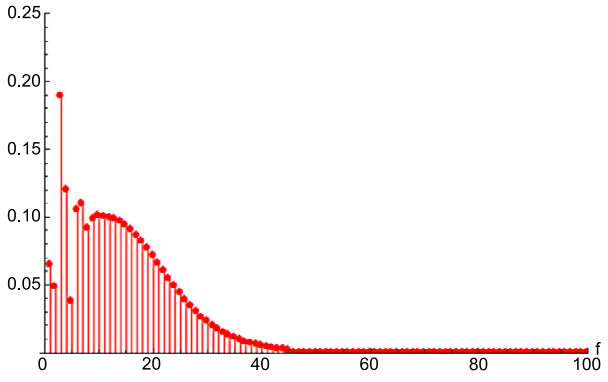

(ii)

Fig. 5. Signal $x(t)$ for Experiment 2. (i) The ECG-like zero mean periodic signal. (ii) Its broad band power spectrum.

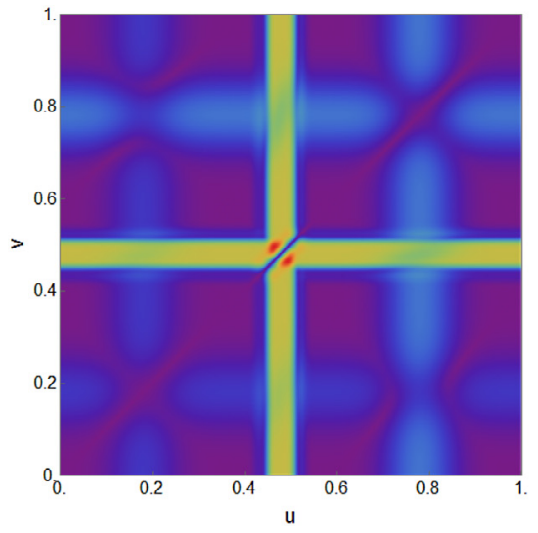

(A)

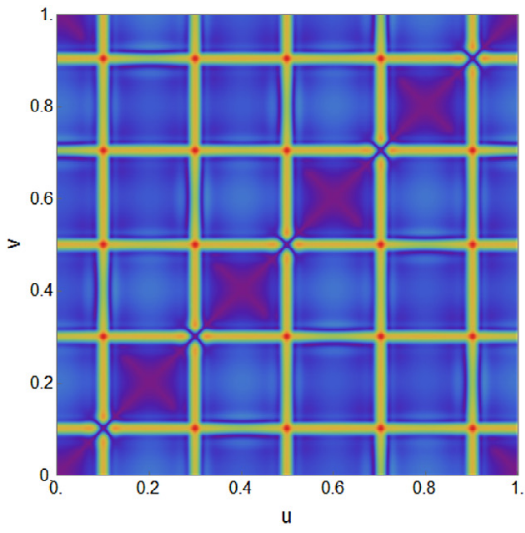

(B)

Fig. 6. URPs for $x(t)$ in Experiment 2. (A) For $M=5$ and $\tau=0.010$. (B) For $M=5$ and $\tau=0.199$.

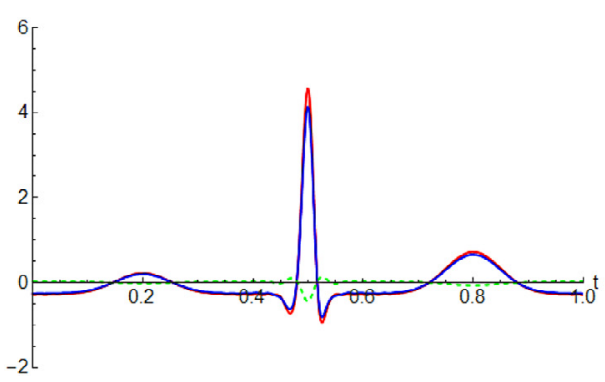

(A)

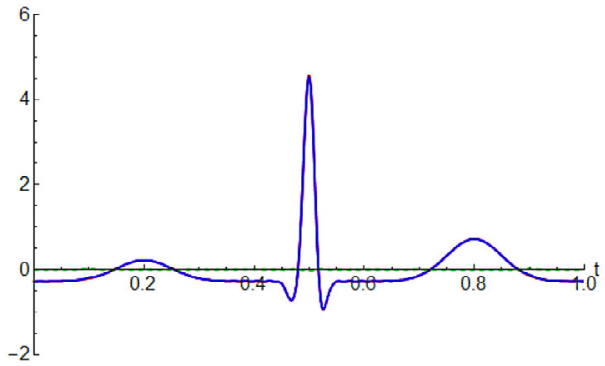

(B)

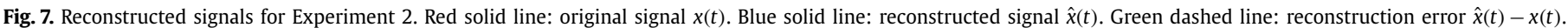
(A) For $M=5$ and $\tau=0.010$. (B) For $M=5$ and $\tau=0.199$. (For interpretation of the colors in this figure, the reader is referred to the web version of this article.)

distributed white noise of zero mean and the small standard deviation $\sigma=0.0006 \max \left(\mathrm{URP}_{X}\right)$. The observed URP again does not visibly differ from the true URP. The reconstructed signal $\hat{x}(t)$ is displayed in Fig. 7(B) (in blue) together with the original signal $x(t)$ (in red, largely overlayed) and the mismatch between both (in green). The corresponding node set $\widehat{K}_{X}$ was in this case obtained as $\widehat{K}_{X}=K_{X} \cup \pm\{195\}$ for which $\hat{h}=1$.

We conclude that the proposed method in both cases allows the signal $x(t)$ to be reconstructed with high accuracy from the lightly disturbed URP. The value $\tau=0.199$ near the unwanted value $\tau=0.200$ does not exhibit any particular difficulties. (In additional experiments, not shown here, we investigated the effect for values of $\tau$ closer to 0.200 by resampling the continuous-time signal $x(t)$ at the corresponding delayed values. This indicated that effects do indeed become significant when $\tau$ approaches 0.200 as expected, but for this to show, the difference should be much smaller than $1 / 1000$.)

\section{Multi-level recurrence plots}

One way to study the information content of RPs is by interpreting them as URPs subjected to (large) disturbances, as induced by the binary discretization resulting from application of the Heaviside function. From this point of view it is natural to also study what happens when a URP is discretized using multiple thresholds instead of just one (as used for RPs). Intuitively, such a multi-level recurrence plot (MRP) may carry far more information than an RP. Depending on the number of threshold levels used, it may also allow for a more accurate reconstruction of the underlying signal. In this section we will briefly sketch such a reconstruction procedure, using the same robust SVD approach described for the 

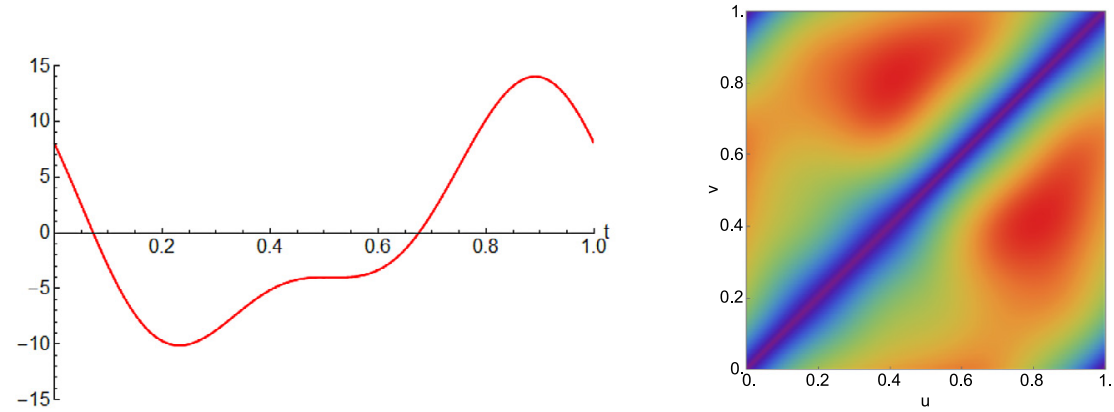

(i) No thresholding
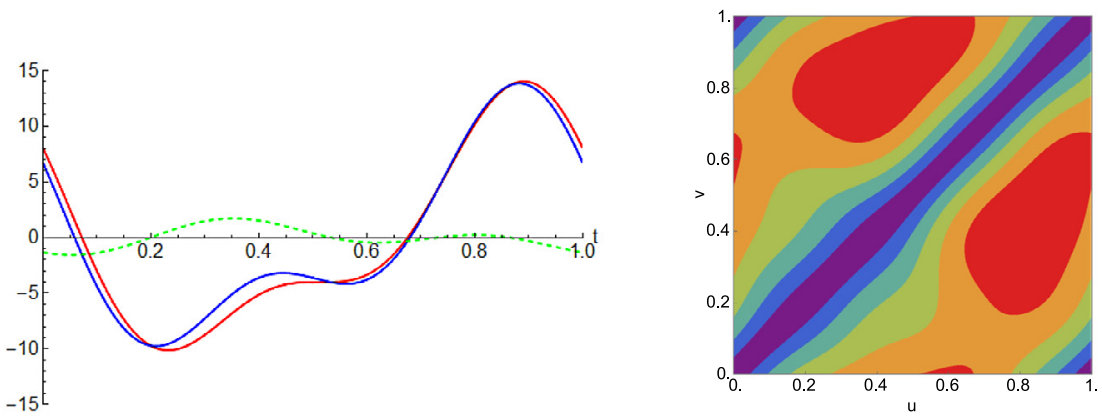

(ii) Five levels
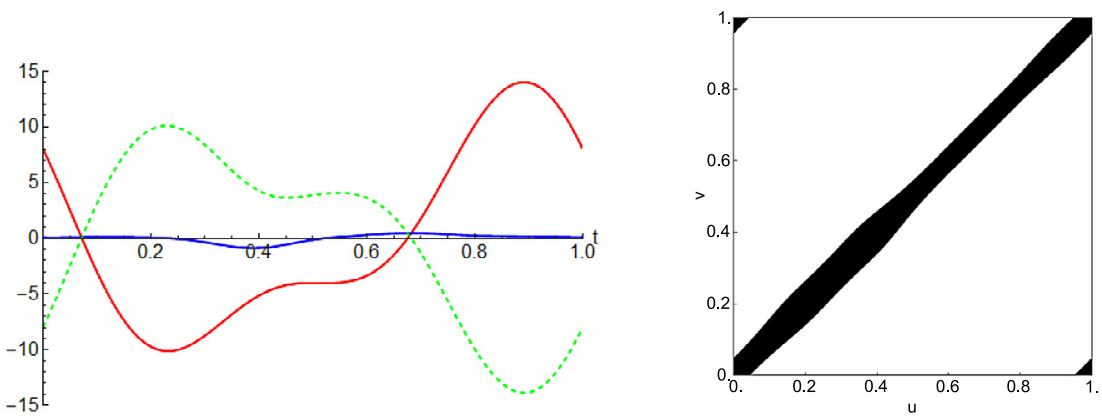

(iii) One level

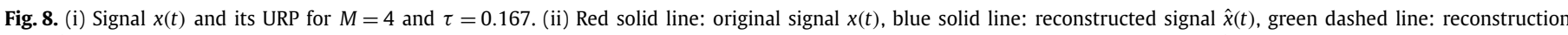

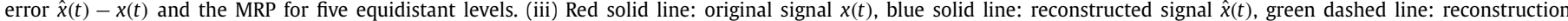
error $\hat{x}(t)-x(t)$ and the RP for just one level. (For interpretation of the colors in this figure, the reader is referred to the web version of this article.)

reconstruction of signals from URPs subject to disturbances. Formally, for given embedding parameters and for $L$ threshold levels $\varepsilon_{1}<\ldots<\varepsilon_{L}$, we introduce the MRP for a signal $x(t)$ as:

$\operatorname{MRP}_{X}(u, v)=\sum_{\ell=1}^{L}\left(\varepsilon_{\ell}-\varepsilon_{\ell-1}\right) \Theta\left(\mathrm{URP}_{X}-\varepsilon_{\ell}\right)$,

where $\varepsilon_{0}:=0<\varepsilon_{1}$ and $\varepsilon_{L}<\max \left(U \mathrm{UP}_{X}\right)$. For example, these levels may be chosen to be equidistant or such that their corresponding level sets partition the square domain $[0,1] \times[0,1]$ of the URP into $L+1$ subsets of equal area. Note that $\operatorname{MRP}_{X}(u, v)=$ $\varepsilon_{1}\left(1-\mathrm{RP}_{X}(u, v)\right)$ for the special case with $L=1$. Also note that an MRP includes the combined information of all the RPs corresponding to the $L$ threshold levels $\varepsilon_{1}, \ldots, \varepsilon_{L}$.

To investigate the information content of an MRP we have carried out two simulation experiments which start from a known zero mean periodic signal $x(t)$ on $[0,1]$. Next we select embedding parameters $M$ and $\tau$, for which we determine the unthresholded recurrence plot URP ${ }_{X}$. Then, for a given set of levels $\varepsilon_{\ell}, \ell \in$ $\{1, \ldots, L\}$ we compute $\mathrm{MRP}_{X}$ from (19). As described in the previous section we reconstruct a signal $\hat{x}(t)$ from $\widehat{U R P}_{X}$.
In our experiments we consider the signal $x(t)=6 \cos (2 \pi t)-$ $8 \sin (2 \pi t)+2 \cos (4 \pi t)-4 \sin (4 \pi t)$, which consists of 1000 samples. The corresponding node set is $K_{X}= \pm\{1,2\}$ with $h=1$. Its URP is generated for the embedding parameters $M=4$ and $\tau=0.167$. For these parameters $h M \tau=0.668 \notin \mathbb{Z}$, so that $G_{X}$ is connected and $x(t)$ is uniquely reconstructible (up to a sign) from $U R P_{X}$. The signal $x(t)$ and the unthresholded recurrence plot $U R P_{X}$ are displayed in Fig. 8(i).

(A) For the five equidistant levels $\varepsilon_{1}, 2 \varepsilon_{1}, 3 \varepsilon_{1}, 4 \varepsilon_{1}, 5 \varepsilon_{1}$ for which $6 \varepsilon_{1}=32.983$ equals the maximum of the URP, the MRP and the reconstructed signal $\hat{x}(t)$ are displayed in Fig. 8(ii) (in blue) together with the original signal $x(t)$ (in red) and the mismatch between both (in green).

(B) For the level $\varepsilon_{1}=5.568$ the RP and the reconstructed signal $\hat{x}(t)$ are displayed in Fig. 8(iii) (in blue) together with the original signal $x(t)$ (in red) and the mismatch between both (in green). Recall that an MRP with one level is related to the corresponding RP as $\operatorname{MRP}_{X}(u, v)=\varepsilon_{1}\left(1-\mathrm{RP}_{X}(u, v)\right)$. This level is chosen such that the recurrence rate (RR or REC) does not exceed $10 \%$, see [3]. The recurrence rate is an RQA quantity which is defined as the percentage of black points in an RP. 
Clearly, the reconstruction $\hat{x}(t)$ of the signal $x(t)$ for five thresholds is better than in the case of only one threshold (or the RP). Both experiments show that the information content of the approximated MRP depends on the number of the thresholds and their values.

\section{Conclusions and discussion}

When recurrence plot analysis is used to investigate properties of signals, through time-delay embedding with parameters $M$ and $\tau$, it is important to know which information is preserved in RPs. For URPs, this question can be answered for signals which have a finite Fourier series representation, as worked out in [1] and Section 2. However, the fact that information is preserved does not mean that it is easily recovered: the inverse problem of computing $x(t)$ from its URP may be ill-conditioned, for instance depending on the choice of embedding parameters. Likewise, for binary RPs, which are obtained from URPs by a simple discretization step, we have that they may also be viewed as the result of a (severe) disturbance applied to the URP. In this paper we have chosen to investigate these issues be studying how well signals can be recovered from disturbed URPs.

In Section 2 we presented a new and explicit characterization of connectedness of $G_{X}$ and, in the course of deriving it, we established that the diameter of a connected graph $G_{X}$ always equals 1 or 2. This new characterization shows for each choice of $M$ precisely which values of $\tau$ will cause disconnectedness. For such values, the URP will not be maximally informative and in choosing the embedding parameters we typically want to avoid them. We illustrated these results with a couple of examples.

In Section 3 it was found that the 2D-Fourier series of $U_{R P}^{2}$ consists of coefficients with a special structure that can be exploited to compute $x(t)$. The coefficients are used to calculate a square matrix $\tilde{I}_{X}$ which is the Hadamard product $\tilde{I}_{X}=W \odot T$ of a rank-one matrix $W$ (determined by the non-zero Fourier coefficients of $x(t)$ only) and a known matrix $T$ (depending entirely on $M$ and $\tau$ ). The matrix $T$ allows for the determination of connectedness of $G_{X}$. If connectedness holds, then, using the property that the diameter is at most $2, T$ can be factored from $\tilde{I}_{X}$ to obtain $W$. With singular value decomposition, the Fourier coefficients of the signal $x(t)$ can then be computed from $W$ in a numerically robust way.

In Section 4 we discussed two simulation experiments to demonstrate this procedure. We briefly analyzed robustness against noise of the reconstruction procedure, by adding noise to a URP before reconstruction. We also investigated sensitivity of the reconstruction near values of $\tau$ for which disconnectedness of $G_{X}$ occurs.

In Section 5 we performed experiments to study the impact of truncation on URPs, such as applied when computing RPs. There we introduced the 'multi-level recurrence plot' (MRP) and we illustrated that an MRP with only a limited number of discretization thresholds can sometimes preserve significantly more information than an RP, while still achieving a high compression rate compared to a URP. In [11] we have shown that knowing the URP along a finite number of well-chosen different curves, one of them being a contour line such as used in constructing an RP, is in general sufficient to determine the URP completely. Intuitively it is likely that a sufficient number of threshold levels provides contours with a similar property (i.e., preserving the information on the signal $x(t)$ up to a sign), motivating the future use of MRPs. When curves are restricted to be diagonals, such as used for analysis in RQA, we expect similar properties to hold as indicated in [11].

Regarding MRPs, a couple of research questions remain open for future investigation. (1) How to select an adequate number of thresholds; and also: which threshold values should be chosen to construct MRPs with maximal information content? (2) To quantify patterns that occur in RPs, several measures have been proposed in the literature, see e.g. [3], that are currently used in RQA. For MRPs an important question concerns the generalization of these measures for use with MRPs.

\section{Appendix A. Proofs}

Proof of Theorem 2.1. (A) Suppose $\tau \notin \mathbb{Q}$. Then for all $(p, q) \in P$ we have $(p-q) M \tau \notin \mathbb{Z}$ so $p$ and $q$ are adjacent. Hence $G_{X}$ is a complete graph (i.e.: connected with $\operatorname{diam}\left(G_{X}\right)=1$ ). See also [1, Corollary 3.5, part (1)].

(B) Suppose $\tau \in \mathbb{Q}$. Then it can be uniquely written as $\tau=\frac{n}{d}$ with $(n, d)$ a co-prime pair of positive integers. (Here $d>1$ and $n \in\{1, \ldots, d-1\}$ because $\tau \in(0,1)$.) For $M \tau$ there also exists a unique representation $M \tau=\frac{m}{\ell}$ with $(m, \ell)$ co-prime. Now $\tau$ can be written as

$\tau=\frac{m}{\ell M}$

Then any pair of nodes $(p, q) \in P$ is non-adjacent iff $\left[\frac{(p-q) n}{d} \notin \mathbb{Z}\right] \wedge$ $\left[\frac{(p-q) m}{\ell} \in \mathbb{Z}\right]$, which equivalently holds iff $\left[\frac{(p-q)}{d} \notin \mathbb{Z}\right] \wedge\left[\frac{(p-q)}{\ell} \in \mathbb{Z}\right]$ because of co-primeness of $(n, d)$ and of $(m, \ell)$.

(B.1) Suppose that $\ell$ does not divide $h$. Then there exists $(u, v) \in P$ for which $u-v$ is not divisible by $\ell$. (If for all $(u, v) \in P$ it held that $\ell$ divides $u-v$, then $\ell$ divided $h$ because of the definition of $h$, contradicting our assumption.) Should $\ell$ divide both $p-u$ and $p-v$ then it also divides their difference $u-v$, which it does not. Hence there is a node $r$ for which $p-r$ is not divisible by $\ell$ (at least one of the two nodes $u$ and $v$ will do), implying that $p$ and $r$ are adjacent. If $(p, q) \in P$ is a non-adjacent pair of nodes, then $\ell$ divides $p-q$. In this case $q-r=(p-r)-(p-q)$ is again not divisible by $\ell$, so that $q$ and $r$ are adjacent. Therefore there is a path of length 2 from $p$ to $q$ via $r$.

It follows that any two nodes are either adjacent or connected by a path of length 2 . I.e., the graph $G_{X}$ is connected and $\operatorname{diam}\left(G_{X}\right) \in\{1,2\}$

(B.2) Suppose that $\ell$ divides $h$. Then denote $f=\frac{h}{\ell}$, whence $h=f \ell$ and $f \in \mathbb{N}$. Also, let $m_{1}$ and $M_{1}$ be the unique positive integers such that $\frac{m}{M}=\frac{m_{1}}{M_{1}}$ with $\left(m_{1}, M_{1}\right)$ co-prime. Note that $\tau=$ $\frac{m}{\ell M}=\frac{m_{1}}{\ell M_{1}}$ with $\left(m_{1}, \ell\right)$ and $\left(m_{1}, M_{1}\right)$ both co-prime, whence also $\left(m_{1}, \ell M_{1}\right)$ is co-prime. Therefore $n=m_{1}$ and $d=\ell M_{1}$.

Because of the definition of $h$, for every $(p, q) \in P$ there exists a positive integer $k$ such that $p-q=k h$. Now $p$ and $q$ are adjacent iff $\left[\frac{k h m_{1}}{\ell M_{1}} \in \mathbb{Z}\right] \vee\left[\frac{k h m}{\ell} \notin \mathbb{Z}\right]$. The latter of these two conditions is never satisfied since $\frac{k h m}{\ell}=k f m$, which is integer. Therefore adjacency holds iff $\frac{k f m_{1}}{M_{1}} \in \mathbb{Z}$, which holds iff $\frac{k f}{M_{1}} \in \mathbb{Z}$ (because $\left(m_{1}, M_{1}\right)$ is co-prime).

(B.2.1) Suppose that $M_{1}$ divides $f$. Then all $(p, q) \in P$ are adjacent and $G_{X}$ is a complete graph.

(B.2.2) Suppose that $M_{1}$ does not divide $f$. Then we may write $\frac{f}{M_{1}}=\frac{g}{M_{2}}$ in a unique way with $\left(g, M_{2}\right)$ co-prime. This also implies that $M_{2}>1$. Then $\frac{k f}{M_{1}}=\frac{k g}{M_{2}}$ is integer iff $\frac{k}{M_{2}}$ is integer, i.e., iff $\frac{p-q}{h M_{2}}$ is integer. So it then holds that $p$ and $q$ are adjacent iff $p$ and $q$ belong to the same equivalence class modulo $h M_{2}$. If there were only one such equivalence class in $K_{X}$, then $h M_{2}$ was a divisor of every value $p-q$ in $D$. Since $M_{2}>1$, this would contradict the definition of $h$. This shows that in $K_{X}$ there are at least two different equivalence classes modulo $h M_{2}$.

Hence, in this case the graph $G_{X}$ is disconnected. The number of different connected components of $G_{X}$ equals the number of (non-empty) equivalence classes of $K_{X}$ modulo $h M_{2}$ and every connected component constitutes a complete subgraph of $G_{X}$. 
Note that $\frac{p-q}{h M_{2}}$ is integer iff $\frac{p-q}{d}$ is integer, since the latter expression is the condition from which the former is derived for the case (B.2.2). Therefore the number of different connected components is also equal to the number of (non-empty) equivalence classes of $K_{X}$ modulo $d$, in agreement with [1, Corollary 3.5, part (5)].

(C) The cases (A), (B.1), (B.2.1) and (B.2.2) are mutually exclusive and cover all possible situations. $G_{X}$ is connected for the cases (A), (B.1) and (B.2.1), with $\operatorname{diam}\left(G_{X}\right) \in\{1,2\}$, which shows statement (2). Also, $G_{X}$ is disconnected if and only if case (B.2.2) applies, i.e.: $\tau$ is rational and $\ell$ divides $h$ and $M_{1}$ does not divide $f$. Then $h M \tau=\frac{h m}{\ell}=f m \in \mathbb{Z}$ which shows that $\tau$ is an integer multiple of $\frac{1}{h M}$. Also $h \tau=\frac{f m}{M}=\frac{f m_{1}}{M_{1}}$ in which $\left(m_{1}, M_{1}\right)$ is co-prime and $M_{1}$ does not divide $f$. Hence $h \tau \notin \mathbb{Z}$ which shows that $\tau$ is not an integer multiple of $\frac{1}{h}$.

Conversely, if $\tau$ is an integer multiple of $\frac{1}{h M}$, then it is rational and there exists a positive integer $n_{1}$ such that $\tau=\frac{n_{1}}{h M}$, whence $M \tau=\frac{n_{1}}{h}$. The definition of $m$ and $\ell$ is such that $\frac{n_{1}}{h}=\frac{m}{\ell}$ with $(m, \ell)$ co-prime. This shows that $\ell$ divides $h$. With $h=f \ell$ we also have $n_{1}=f m$. Now $\tau=\frac{f m}{h M}=\frac{f m_{1}}{h M_{1}}$ with $\left(m_{1}, M_{1}\right)$ co-prime. Then such $\tau$ is not an integer multiple of $\frac{1}{h}$ iff $\frac{f m_{1}}{M_{1}}$ is not integer, iff $M_{1}$ does not divide $f$. Thus case (B.2.2) applies and $G_{X}$ is disconnected. This shows that $G_{X}$ is disconnected iff $[h M \tau \in \mathbb{Z}] \wedge[h \tau \notin \mathbb{Z}]$, which proves statement (1).

Proof of Proposition 3.1. Using the fact that $X(t)$ is real, it follows that $\operatorname{URP}_{X}^{2}(u, v)=\langle X(u)-X(v), X(u)-X(v)\rangle=I_{X}(u, u)-$ $2 I_{X}(u, v)+I_{X}(v, v)$. The 2D-Fourier coefficients of the term $-2 I_{X}(u, v)$ follow from Eqn. (6). Note that $I_{X, p, 0}=I_{X, 0, q}=0$ for all $p$ and $q$, because $c_{0}=0$.

Also, $I_{X}(u, u)=\sum_{p \in \mathbb{Z}} \sum_{q \in \mathbb{Z}} c_{p} \overline{c_{q}} e^{2 \pi(p-q) u i}\left\langle T_{p}, T_{q}\right\rangle$, in which $\left\langle T_{p}, T_{q}\right\rangle=\left\langle T_{p-q}, T_{0}\right\rangle$. Changing the summation variables from $(p, q)$ to $(r, n)=(p-q, p)$, it follows that $I_{X}(u, u)=$ $\sum_{r \in \mathbb{Z}}\left(\sum_{n \in \mathbb{Z}} c_{n} \overline{c_{n-r}}\right) e^{2 \pi r u i}\left\langle T_{r}, T_{0}\right\rangle$. Hence, its 2D-Fourier coefficients only contribute to $C_{X, r, 0}$, by the amount $\left\langle T_{r}, T_{0}\right\rangle \sum_{n \in \mathbb{Z}} c_{n} \overline{c_{n-r}}$. The contribution of the term $I_{X}(v, v)$ is computed likewise to involve only the coefficients $C_{X, 0, r}$ by similar amounts.

To obtain the given expressions for $C_{X, p, q}$ in terms of the coefficients $I_{X, p, q}$, note that $\sum_{n \in \mathbb{Z}} I_{X, p-n, n}=\sum_{n \in \mathbb{Z}} c_{p-n} \overline{c_{-n}}\left\langle T_{p-n+n}, T_{0}\right\rangle$. With $c_{k}=\overline{c_{-k}}$ for a real signal, the expression for the case $p \neq 0$ and $q=0$ follows. The other cases are obtained likewise.

Finally it is remarked that the coefficients $C_{X, p, q}$ are welldefined, because for every $r \in \mathbb{Z}$ the quantity $\sum_{n \in \mathbb{Z}} c_{n} \overline{c_{n-r}}$ converges, since we are dealing with $L^{2}$-functions on $[0,1)$.

Proof of Proposition 3.2. (1) Clearly, $J$ equals its own inverse as a result of its definition. Since every permutation is orthogonal, $J^{-1}=J^{T}$.

(2) Because $c_{-k}=\overline{c_{k}}$, the definition of $J$ is such that $J w=\bar{w}$. Then $w=J \bar{w}$ by part (1).

(3) Let $x$ be an eigenvector of $W$ with eigenvalue $\lambda$, whence $w w^{*} x=\lambda x$. Using parts (1) and (2) it holds that $J w w^{*} J J x=\lambda J x$ and equivalently $\bar{w}^{*} J x=\lambda J x$. Because every eigenvalue of a Hermitian matrix is real, $\lambda$ is real. Complex conjugation therefore gives: $w w^{*} J \bar{x}=\lambda J \bar{x}$. Finally, note that $J \overline{(J \bar{x})}=x$.

Proof of Proposition 3.3. Because $W$ is Hermitian and positive semi-definite and nonzero (hence of rank one), $\lambda$ is the unique eigenvalue of $W$ which is real and positive. The vector $u$ is a corresponding normalized eigenvector of $W$, which determined up to a unimodular scalar factor. The vector $w$ is also an eigenvector, with eigenvalue $w^{*} w$. Hence $\lambda=w^{*} w$ and $w=\alpha \sqrt{\lambda} u$ for some unimodular scalar $\alpha$.
Now: $w=J \bar{w}$ gives $\alpha \sqrt{\lambda} u=\bar{\alpha} \sqrt{\lambda} J \bar{u}$, which is equivalent to $\alpha^{2} u=J \bar{u}$, whence $\alpha^{2}=u^{*} J \bar{u}$. This shows that $\alpha= \pm\left(u^{*} J \bar{u}\right)^{1 / 2}$ which fixes $\alpha$ and $w$ up to a sign \pm 1 , as claimed.

Proof of Theorem 3.4. Note that $\left(\hat{I}_{X}\right)_{k, k}=M\left|c_{k}\right|^{2}$. Since the indices $k$ are restricted to $K_{X}$, this is strictly positive and $\operatorname{diag}\left(\hat{I}_{X}\right)^{-1}$ exists. The entries of $\hat{I}_{X} \operatorname{diag}\left(\hat{I}_{X}\right)^{-1} \hat{I}_{X}$ are given by:

$$
\begin{aligned}
\left(\hat{I}_{X} \operatorname{diag}\left(\hat{I}_{X}\right)^{-1} \hat{I}_{X}\right)_{p, q} & =\sum_{k \in K_{X}} \frac{\left(\hat{I}_{X}\right)_{p, k}\left(\hat{I}_{X}\right)_{k, q}}{\left(\hat{I}_{X}\right)_{k, k}} \\
& =\sum_{k \in K_{X}} \frac{c_{p} \overline{c_{k}} S_{p, k} c_{k} \overline{c_{q}} S_{k, q}}{c_{k} \overline{c_{k}} S_{k, k}} \\
& =\frac{c_{p} \overline{c_{q}}}{M} \sum_{k \in K_{X}} S_{p, k} S_{k, q} .
\end{aligned}
$$

Here $c_{p} \overline{c_{q}}=W_{p, q}$ and $\sum_{k \in K_{X}} S_{p, k} S_{k, q}=\left(S^{2}\right)_{p, q}$. This proves Eqn. (15).

Finally, note that the entry $\left(S^{2}\right)_{p, q}=\sum_{k \in K_{x}} S_{p, k} S_{k, q}$ is composed of a sum of products $S_{p, k} S_{k, q}=\left|\left\langle T_{p}, T_{k}\right\rangle\left\langle T_{k}, T_{q}\right\rangle\right|$. Each such term is non-zero if and only if in the graph $G_{X}$ there is a path of length 2 from node $p$ to node $q$ via node $k$, and by construction the term then is positive. Therefore, $\left(S^{2}\right)_{p, q}>0$ if and only if there is a path of length 2 from $p$ to $q$. In this formulation also adjacency of any node $p$ to itself is included, which is supported by $\left\langle T_{p}, T_{p}\right\rangle=M>0$. Therefore, all the entries of $S^{2}$ are strictly positive if and only if the diameter of $G_{X}$ is either 1 or 2 . In Theorem 2.1 this was proven to hold for $G_{X}$ if it is connected.

Proof of Proposition 3.5. First note that the given bound $\delta$ is $\leq 1$ for all $d \geq 2$, with equality for $d=2,3$, 4. If $(p-q) \tau \in \mathbb{Z}$ then $S_{p, q}=M$ and so the claim of part (1) holds. For $S_{p, q}=0$ we equivalently have that $(p-q) M \tau$ is integer while $(p-q) \tau$ is not; this case does not need to be addressed. It follows that we must still prove the claim of part (1) for $(p-q) M \tau$ not being integer. The expression for $S_{p, q}$ only depends on $k=p-q$ and with $\tau=\frac{n}{d}$ it takes the form

$f(k)=\frac{\left|\sin \left(\frac{\pi M k n}{d}\right)\right|}{\left|\sin \left(\frac{\pi k n}{d}\right)\right|}$,

which we aim to analyze for integer $k$ such that both the numerator and the denominator of $f(k)$ are nonzero.

Clearly a lower bound for $f(k)$ is obtained when the numerator is as small as possible and the denominator as large as possible. Writing $M k n=\ell d+r$ with $\ell$ integer and $r \in 0,1, \ldots, d-1$, shows that $r=0$ is excluded to avoid a zero value for the numerator. Then a lower bound for the numerator is obtained for $r=1$ (as well as for $r=d-1$ ). For the denominator an obvious upper bound is given by 1 . Together this gives the lower bound

$$
f(k) \geq \sin \left(\frac{\pi}{d}\right) .
$$

To refine this bound, first note that the upper bound 1 on the denominator is achieved if and only if $\frac{k n}{d}$ is an integer plus a half. Then the numerator equals $\left|\sin \left(\frac{\pi M}{2}\right)\right|$ which is either 0 or 1 . As we excluded zero values for the numerator from consideration, we find that in this case $f(k)=1$. Note that for $d=2$ this case occurs for all $k$ considered and the bound $\delta$ given in part (1) applies.

If the upper bound 1 on the denominator is not achieved, then a new upper bound is given for $k$ such that $\frac{k n}{d}$ is as close as possible to an integer plus a half. If $d$ is odd, the difference is at least 
$\frac{1}{2 d}$. Then the denominator has the upper bound $\sin \left(\frac{\pi}{2}-\frac{\pi}{2 d}\right)=$ $\cos \left(\frac{\pi}{2 d}\right)$, for which:

$f(k) \geq \frac{\sin \left(\frac{\pi}{d}\right)}{\cos \left(\frac{\pi}{2 d}\right)}=2 \sin \left(\frac{\pi}{2 d}\right)$,

as claimed in part (1). If $d$ is even, hence $d \geq 4$, the difference is at least $\frac{1}{d}$. Now the denominator has the upper bound $\sin \left(\frac{\pi}{2}-\frac{\pi}{d}\right)=$ $\cos \left(\frac{\pi}{d}\right)$, for which:

$f(k) \geq \frac{\sin \left(\frac{\pi}{d}\right)}{\cos \left(\frac{\pi}{d}\right)}=\tan \left(\frac{\pi}{d}\right)$,

as claimed in part (1) too.

For part (2), observe that $\left(S^{2}\right)_{p, q}=\sum_{k \in K_{X}} S_{p, k} S_{k, q}$. In view of Theorem 2.1 and because each term in this sum is nonzero if and only if there is a path of length 2 from $p$ to $q$ via $k$, together with the bound of part (1) the claim of part (2) follows.

\section{References}

[1] A. Sipers, P. Borm, R. Peeters, Phys. Lett. A 375 (2011) 2309.

[2] J.-P. Eckmann, S. Oliffson Kamphorst, D. Ruelle, Europhys. Lett. 4 (1987) 973.

[3] N. Marwan, M.C. Romano, M. Thiel, J. Kurths, Phys. Rep. 438 (2007) 237.

[4] F. Takens, Detecting strange attractors in turbulence, in: D. Rand, L. Young (Eds.), Dynamical Systems and Turbulence, in: Lecture Notes in Mathematics, vol. 898, Springer, Berlin, 1981, pp. 366-381.

[5] L. Ljung, System Identification: Theory for the User, PTR Prentice Hall Information and System Sciences Series, 1991.

[6] D.S. Broomhead, G.P. King, On the qualitative analysis of experimental dynamical systems, in: S. Sarkar (Ed.), Nonlinear Phenomena and Chaos, Adam Hilger, 1986, pp. 113-144.

[7] M. Thiel, M.C. Romano, J. Kurths, Phys. Lett. A 330 (2004) 343.

[8] Y. Hirata, S. Horai, K. Aihara, Eur. Phys. J. Spec. Top. 164 (2008) 13

[9] G. Robinson, M. Thiel, Chaos 19 (2009) 023104.

[10] W. Rudin, Real and Complex Analysis, McGraw-Hill, 1987.

[11] A. Sipers, P. Borm, R. Peeters, Quantifying redundancy and information content of lines in recurrence plots using the theory of framework rigidity, in: N. Marwan, M. Riley, A. Giuliani, C.L. Webber Jr. (Eds.), Translational Recurrences, in: Springer Proceedings in Mathematics \& Statistics, vol. 103, Springer International Publishing, 2014, pp. 55-73. 